\title{
Electron Tomography of Meiotic Spindle Components in Caenorhabditis elegans
}

\author{
Eileen T. O’Toole*, Martin Srayko**, Jana Mäntler**, and Thomas Müller-Reichert**
}

*Boulder Laboratory for 3-D Electron Microscopy of Cells, University of Colorado, Boulder, CO 80309

**Max Planck Institute of Molecular Cell Biology and Genetics, Electron Microscopy Facility, D01307 Dresden, Germany

In mitosis, pole-proximal microtubule ends are organized around centrosomes consisting of a pair of centrioles and pericentriolar material. In C. elegans mitotic centrosomes contain two types of poleproximal ends. Roughly $80 \%$ of these ends are closed (i.e. capped), whereas $\sim 20 \%$ are open [1]. In contrast, pole-proximal ends in female meiosis are arranged in acentrosomal spindle poles that lack typical centrosome components. We were interested in applying tomographic reconstruction to visualize the organization and end morphology of microtubules in an acentrosomal context.

For the preparation of whole worms, we have applied a combination of methods, including highpressure freezing and freeze substitution (HPF/FS). Material prepared by HPF/FS was subsequently thin-layer embedded, allowing us to select worms prior to serial sectioning [2]. To obtain highresolution 3-D structure data, serial $300 \mathrm{~nm}$ thick sections containing meiotic spindles were imaged in an IVEM (TECNAI F30, FEI), and tilted images were collected about two orthogonal axes. Tomographic reconstructions were calculated using back projection algorithms and combined using the method of Mastronarde [3].

Electron tomographic reconstruction allowed us to trace individual microtubules in 3-D and to identify pole-proximal microtubule ends in wild-type specimens (Fig. 1). Spindle microtubules were not focused at a particular region of the meiotic chromosomes. Analysis of the tomograms revealed that the majority of pole-proximal microtubule ends were open and the three-dimensional models show a statistical distribution of these open, pole-proximal ends within the spindle. Strikingly, 20$40 \%$ of the spindle microtubules showed evidence of fragmentation. In contrast, tomograms of spindles from a MEI-1 (katanin-like protein) loss-of-function mutant [4] revealed disorganized spindles and no evidence of microtubule fragmentation.

As will be discussed, these results are thought to reflect: first, chromatin-induced nucleation or capture of microtubules and second, microtubule-severing activity required in meiotic spindles to keep microtubules short.

References:

[1] E.T. O’Toole et al., J. Cell Biol. (2003) 451-456.

[2] T. Müller-Reichert et al., J. Microsc. (2003) 71-80.

[3] D.N. Mastronarde, J. Struct. Biol. (1997) 343-352.

[4] M. Srayko et al., Genes Dev. (2000) 1072-1084. 


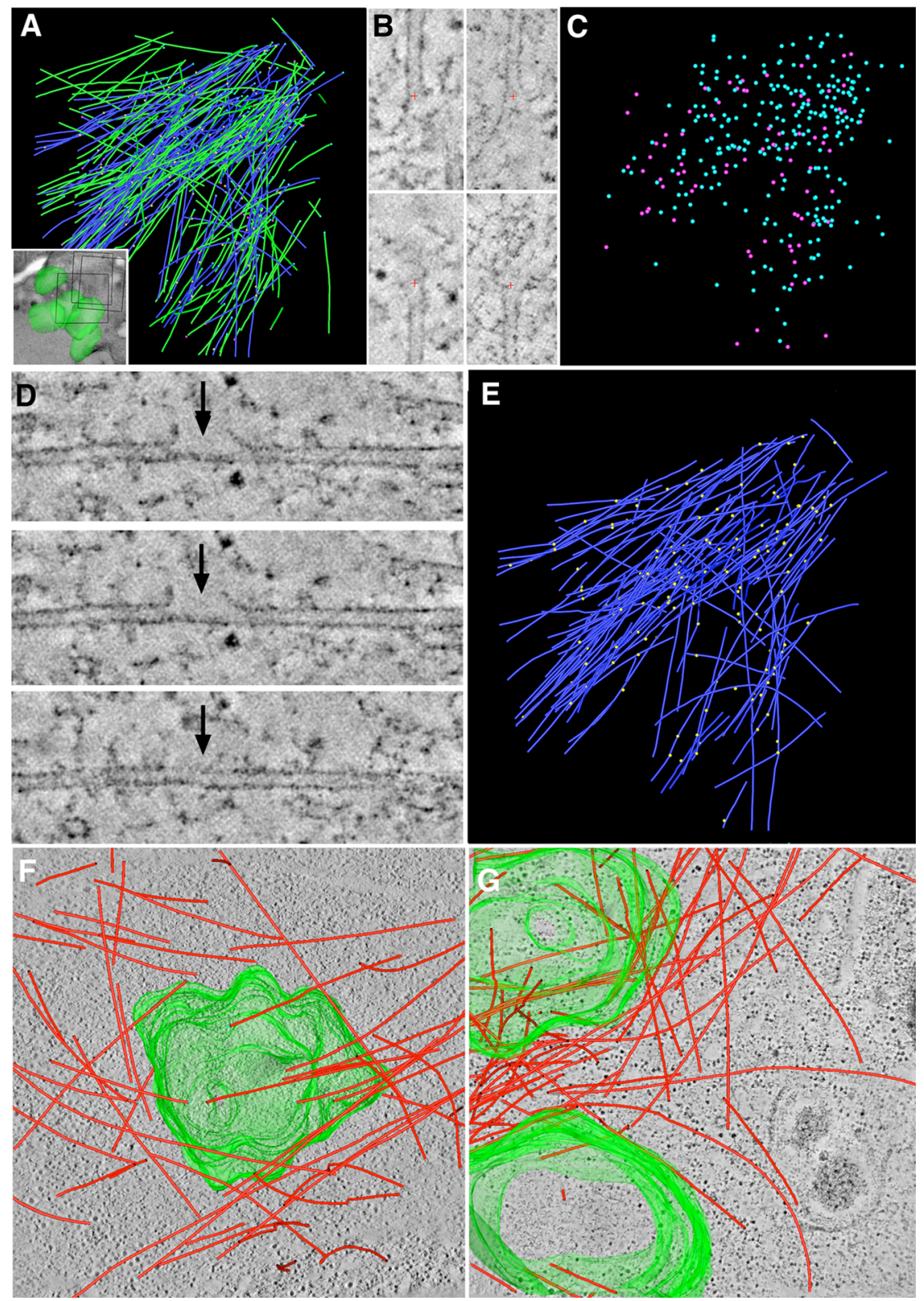

Figure 1. Distribution of microtubules in a meiotic spindle pole. (A) Portion of a C. elegans meioitic spindle. Model was built from three serial tomograms (inset). Microtubules (blue, green lines) form a short, barrel-shaped spindle. (B) Microtubule ends of pole-proximal (top) as well as pole-distal ends (bottom) are open. (C) Microtubule pole-proximal ends (blue spheres) are not focused at a distinct pole but are distributed throughout the spindle. Microtubule pole-distal ends (purple spheres) were also found throughout the spindle as well as at the chromosomes. (D) Selected, serial $2 \mathrm{~nm}$ tomographic slices were rotated to view a microtubule along its length. Evidence of a lateral disruption of the microtubule is evident (arrow). (E) Model showing fragmented microtubules (blue lines). Some microtubules showed numerous points of fragmentation, as indicated by yellow spheres. $(\mathrm{F}, \mathrm{G})$ MEI-1 loss-of-function mutant showed disorganized microtubules (red) around meiotic chromosomes (green) and no evidence of microtubule fragmentation. 